\title{
http://www.unibe.ac.cr/ojs/index.php/psicoinnova
}

\section{Intensidad de uso de Facebook y su relación con el auto-concepto social}

\section{David Torres \\ Universidad de Iberoamerica}

\section{Resumen}

Diversos estudios han evaluado las relaciones entre el uso de redes sociales y el autoconcepto en diversas poblaciones a nivel mundial (Gangadharbatla, 2008; Gonzales \& Hancock, 2011; Kujath, 2011; Ledbetter et al., 2011; Orr et al., 2009; Valkenburg \& Peter, 2007b; Valkenburg \& Peter, 2007a; Valkenburg, Peter, \& Schouten, 2006). El presente artículo sigue esa línea al evaluar, en dos estudios, esta relación con mediciones también de habilidades sociales, satisfacción percibida con las relaciones interpersonales y la cantidad de amigos en línea. El primer estudio contó con la participación de 164 adolescentes con edades entre edades los 15 y 17 años $(\mathrm{M}=15.12$, $\mathrm{DE}=0.71)$, encontrándose que el autoconcepto social puede ser predicho por la satisfacción con las relaciones interpersonales, las habilidades sociales y la cantidad de tiempo que se permanece en Facebook. Por su parte, el segundo estudió contó con la participación de 225 estudiantes universitarios con edades entre los 18 y 31 años $(\mathrm{M}=22.03, \mathrm{DE}=2.97)$ y se encontró que el auto-concepto social puede ser predicho por la satisfacción con las relaciones interpersonales y las habilidades sociales. Las implicaciones de ambos estudios son discutidas.

Palabras Clave: Facebook, Auto-concepto, Adolescentes, Adultez joven

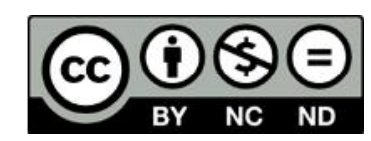




\section{Introducción}

La comunicación mediada por computadora (CMC) tiene como una de sus características el dar oportunidades de interacción a personas a las que el contacto interpersonal se ve dificultoso debido a inhibiciones sociales, en ésta, las personas con mayores niveles de timidez y sociabilidad perciben más cercanas y satisfactorias las relaciones en línea que quienes reportan menores valores de estos constructos (Sheeks \& Birchmeier, 2007). Además, este tipo de comunicación aumenta la conciencia de sí mismo y las auto-revelaciones y disminuye la influencia interpersonal, de forma tal que en la CMC se revela más información personal que cuando se comunica cara a cara (Joinson, 2001; Sassenberg, Boos, \& Radung, 2005).

Se ha encontrado que las relaciones cara a cara son consideradas como relaciones con mayores grados de profundidad, amplitud, compromiso, códigos de cambio, entendimiento, interdependencia y convergencia de redes que las relaciones en línea, sin embargo estas diferencias se ven disminuidas conforme avanza el tiempo en la relación. Estos resultados sirven para aportar evidencias a la hipótesis que plantea que la comunicación mediada por computadora dificulta el desarrollo de relaciones cercanas debido a la falta de claves de contexto social y lenguaje no verbal hace, sin embargo, esta hipótesis no considera el tiempo de la relación (Chan \& Cheng, 2004).

Centrándose en las redes sociales, Facebook se ha difundido enormemente en los últimos años. Esta red, creada por Mark Zuckenberg en el año 2004, ha crecido exponencialmente con el paso de los años alcanzado los 500 millones de usuarios en sólo 6 años de existencia, convirtiéndose en la red social con mayor penetración a nivel mundial y 
una de las tres marcas en línea más populares y el sitio web en el que más tiempo permanecen los usuarios (Nielsen, 2010).

Las principales motivaciones por las que se unen las personas a dicha red son por la sugerencia de amigos, porque una gran parte de su círculo social es usuario de Facebook o para ponerse en contacto con personas conocidas. Siendo estas también algunas de las razones por las que se mantienen en esta red, además de la sociabilización (hacer nuevos amigos), moda, pasatiempo, búsqueda de afecto y obtener información social (noticias, reuniones sociales) (Quan-Haase \& Young, 2010; Raacke \& Bonds-Raacke, 2008; Sheldon, 2008b).

En la misma línea, se ha encontrado que las personas que utilizan Facebook para pasar el tiempo y sentirse menos solos son las personas que presentan mayores niveles de ansiedad y miedo en las comunicaciones cara a cara, sin embargo tienen también menos amigos en dicha red social, sin embargo, las evidencias muestran que las personas que se involucran en relaciones interpersonales en línea son las que están más dispuestas a comunicarse en la vida cotidiana (Sheldon, 2008a).

Se tiene además que, en su mayoría, las personas utilizan las redes sociales (Facebook y MySpace) como un complemento de la interacción cara a cara, más allá que como un sustituto de la misma (Kujath, 2011). Resultados que se complementan con estudios que muestran que tanto el uso de Facebook como de la comunicación cara a cara predicen positivamente la cercanía en las relaciones (Ledbetter et al., 2011). Estos resultados apoyan la hipótesis llamada "el rico se hace más rico" que sostiene que son, principalmente, las 
computadora; a la vez se muestra evidencia contraria a la hipótesis de la compensación social, la cual propone que son las personas solitarias o con problemas de ansiedad social y dificultades para desarrollar amistades en la vida real los que buscan la comunicación en línea (Valkenburg \& Peter, 2007b).

Aún así, es posible encontrar resultados que favorecen la hipótesis de compensación social, como los que muestran que las personas con mayores niveles de timidez tienden a permanecer durante más tiempo en Facebook que quienes muestran bajos niveles de ansiedad, sin embargo, estos también son los que tienen menos amigos en la red social. Por otro lado, mientras más positivas sean las actitudes hacia el uso de Facebook, hay un mayor tiempo de permanencia en la misma red social (Orr et al., 2009).

Por otro lado, investigaciones recientes muestran que el uso de redes sociales puede favorecer el auto-concepto social en los adolescentes con sólo recibir comentarios positivos o negativos en sus perfiles, actualizaciones de estado o comentarios de forma que comentarios positivos favorecerán el auto-concepto mientras que comentarios negativos lo disminuirán (Valkenburg et al., 2006). En la misma línea, el sólo hecho de exponerse a la información brindada en el propio perfil de Facebook favorece el auto-concepto, en comparación con verse en el espejo o no hacer nada (Gonzales \& Hancock, 2011).

De la misma forma, estudios de conductancia de la piel han encontrado que los usuarios muestran mayores respuestas emocionales placenteras mientras extraen información social en esta red (visitar los perfiles de los amigos) que cuando realizan búsquedas sociales superficiales (revisar las publicaciones recientes y cambios de estado), mostrando que hay 
una alteración de los estados emocionales mientras se realiza una conexión con los amigos en línea, lo cual se puede explicar como una activación motivacional hacia la búsqueda de dicha información (Wise, Alhabash, \& Park, 2010).

Así mismo, se ha encontrado una relación entre actitudes positivas hacia las redes sociales y el auto-concepto colectivo, así como con la necesidad de pertenencia. (Gangadharbatla, 2008).

Contextualizando, en Costa Rica un $95 \%$ de los usuarios web poseen una cuenta en por lo menos una red social (Vindas, 2010), siendo Facebook la red social preferida $(92,1 \%)$. Actualmente, hay 1935740 de usuarios costarricenses registrados en Facebook, lo que significa una penetración del $42.86 \%$ de la población total del país y de un $96.79 \%$ de la población usuaria de Internet (Socialbakers, 2012).

El presente artículo presenta dos estudios que pretenden indagar sobre el papel que juega la red social Facebook en las relaciones interpersonales, específicamente en el autoconcepto social.

Es necesario considerar en este punto que se han encontrado diferencias en el papel que juega la comunicación en línea en el bienestar percibido, dependiendo de si ésta se realiza con conocidos o con extraños (Valkenburg \& Peter, 2007a), por esto se tomó en cuenta la cantidad de amigos que se poseen en la red social, así como el porcentaje de ellos que conoce personalmente .

De igual forma se consideraran como variables importantes la intensidad de uso de la misma red social, la satisfacción percibida con las relaciones interpersonales mediadas por 
entre sexos.

Basándose en lo descrito anteriormente se busca con este estudio responder a preguntas como ¿Qué papel juega la intensidad de uso de Facebook en el auto-concepto social?, ¿Existen diferencias en el auto-concepto social entre altos y bajos usuarios de Facebook?, ¿Qué papel juega el género en la relación entre la intensidad de uso de Facebook y el auto-concepto social?

Cada uno de los estudios fue realizado con dos poblaciones diferentes, el primer estudio fue realizado con adolescentes mientras que el segundo fue llevado a cabo con estudiantes universitarios. Sin embargo, para ambos estudios se midieron las mismas variables y se plantearon las mismas hipótesis, las cuáles son:

H1: Una alta satisfacción con las relaciones interpersonales y habilidades sociales están relacionadas con mayores niveles de auto-concepto social.

H2: Existen diferencias entre altos y bajos usuarios de Facebook con respecto a la relación entre la satisfacción con las relaciones interpersonales, habilidades sociales y auto-concepto social.

H3: Existen diferencias por sexo con respecto a relación entre la satisfacción con las relaciones interpersonales, habilidades sociales y auto-concepto social.

H4: La cantidad de amigos conocidos cara a cara tiene una influencia en las relaciones mencionadas anteriormente. 
Estudio 1: Intensidad de uso de Facebook y su relación con el auto-concepto social en adolescentes

Método

Participantes

Para el presente estudio se contó con la participación de 164 estudiantes de colegio con edades entre los 15 y 17 años $(M=15.12, D E=0.71)$, de los cuales 87 eran mujeres y 77 hombres, además 88 estudiaban en colegios públicos y 76 privados.

\section{Instrumentos}

Con el fin de medir el auto-concepto social se utilizó la sub-escala de auto concepto social del Perfil de auto-percepción de Harter (Van-der-Bergh \& Marcoen, 1999), la cual consta de 6 ítems. Esta escala funciona como una medida de auto-reporte valorada mediante una escala tipo Likert en un rango de uno (totalmente en desacuerdo) a siete (totalmente de acuerdo), con una buena consistencia interna $(\alpha=.82)$. Algunos ítems de la misma son "soy popular" y "me es fácil hacer amigos".

Para medir habilidades sociales se utilizó la versión modificada de Pérez (sin publicar, en (Brenes, 2009)) de la escala original de timidez de Leary. Ésta es un autoreporte unidimensional de 13 ítems $(\alpha=.74)$ valorado mediante una escala tipo Likert en un 
ejemplos de ítems de esta escala son los 38 siguientes: "Me siento tenso cuando estoy con gente que no conozco bien”, "Me resulta fácil preguntarle información a la gente".

Por otro lado, la satisfacción percibida con las relaciones interpersonales fue como una relación inversa con la soledad por lo que se utilizó la Escala Revisada de Soledad de UCLA (RULS; Russell, Peplau y Cutrona, 1980; en Torres (2009)), escala de 20 ítems que se evalúa con valores del 1 al 4, donde a mayor valor mayor frecuencia. La consistencia interna de esta escala es aceptable $(\alpha=.76)$. Los valores de esta escala fueron revertidos para obtener un valor de satisfacción percibida con las relaciones interpersonales. Algunos ejemplos de esta escala son "Me falta compañía", "No tengo a nadie cerca de mî".

La intensidad de uso de Facebook se midió a través de 4 preguntas en una escala tipo Likert de 8 puntos, donde se preguntó sobre la cantidad de veces al día que se ingresa a Facebook, qué tanto tiempo permanece en dicha página, cada cuánto comenta los estados, publicaciones o fotografías de amigos y páginas a las que está asociado, y cada cuánto tiempo actualiza su perfil de Facebook. Además, para medir la cantidad de amigos y amigas en Facebook se preguntó por la cantidad de amigos y amigas que se poseen en la red social Facebook, así como la cantidad aproximada de los mismos que conoce cara a cara.

Por último, se preguntaron datos como edad, sexo, colegio de procedencia y otras variables para medir la condición socioeconómica. 


\section{Procedimiento}

Como un primer paso, para comprobar la validez de los instrumentos se realizó una prueba piloto con 80 estudiantes de colegio. Luego, la recolección de datos definitiva se realizó con cuestionarios de papel y lápiz y su llenado fue de forma voluntaria.

\section{Resultados}

Para responder a las preguntas planteadas se realizó un análisis de correlación para determinar las relaciones entre el auto-concepto social y las demás variables en estudio, seguido a esto se realizaron análisis de regresión múltiples para determinar los predictores del auto-concepto social.

El análisis de correlación simple mostró relaciones positivas entre el auto-concepto social y las habilidades sociales $(r=.40, p<.001)$, la satisfacción percibida con las relaciones interpersonales $(r=.48, p<.001)$, la cantidad de amigos y amigas que se tiene en Facebook ( $r=.24, p=.003)$, la cantidad de estos amigos y amigas que conoce personalmente $(r=.23$, $p=.005)$, cada cuanto tiempo ingresa a Facebook $(r=.17, p=.003)$, el tiempo que se permanece en Facebook ( $r=.27, p=.001)$ y cada cuanto tiempo comenta los perfiles de sus amigos (as) o las páginas a las que se encuentran asociados (as) $(r=.19, p=.018)$. No se encontraron relaciones significativas con sexo $(r=.02, p=.830)$, edad $(r=.-.09, p=.284)$, ni la cantidad de veces que actualiza su perfil de Facebook $(r=.07, p=.388)$ (Tabla 1).

Tabla 1. Medias, desviaciones típicas y correlaciones simples de las variables en estudio en adolescentes.

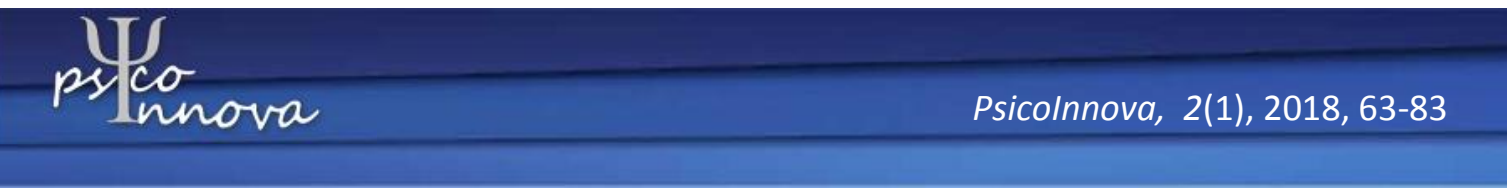




\begin{tabular}{|c|c|c|c|c|c|c|c|c|c|c|c|c|c|}
\hline & $\alpha$ & M & $\mathrm{DE}$ & 2 & 3 & 4 & 5 & 6 & 7 & 8 & 9 & 10 & 11 \\
\hline $\begin{array}{l}\text { 1. Auto-concepto } \\
\text { social }\end{array}$ & .79 & 4.96 & 1.25 & $.40^{* *}$ & $.48^{* *}$ & -.09 & .02 & $.24^{*}$ & $.23^{*}$ & $.17^{*}$ & $.27^{*}$ & $.19^{*}$ & .07 \\
\hline 2. Habilidades sociales & .74 & 5.05 & .95 & - & $.45^{* *}$ & -.02 & .02 & $.22^{*}$ & $.24^{*}$ & .08 & .15 & .02 & .12 \\
\hline $\begin{array}{l}\text { 3. Satisfacción } \\
\text { percibida }\end{array}$ & .76 & 5.21 & .84 & & - & -.10 & .12 & $.25^{*}$ & $.27^{*}$ & $.24^{*}$ & $.17^{*}$ & $.26^{*}$ & $.20^{*}$ \\
\hline 4. Edad & - & 15.12 & .71 & & & - & $\begin{array}{c}- \\
.00\end{array}$ & .07 & .07 & .06 & -.05 & .14 & -.04 \\
\hline 5. Sexo & - & - & - & & & & - & $.32^{*}$ & $.26^{*}$ & $.31^{*}$ & $.19^{*}$ & $.23^{*}$ & .13 \\
\hline $\begin{array}{l}\text { 6. Amigos en } \\
\text { Facebook }\end{array}$ & - & 528.49 & 505.80 & & & & & - & $.84^{*}$ & $.34^{*}$ & $.33^{*}$ & $.28^{*}$ & $.23^{*}$ \\
\hline $\begin{array}{l}\text { 7. Amigos que conoce } \\
\text { personalmente }\end{array}$ & - & 279.92 & 254.68 & & & & & & - & $.33^{*}$ & $.31^{*}$ & $.30^{*}$ & .15 \\
\hline $\begin{array}{l}\text { 8. Frecuencia que } \\
\text { ingresa a Facebook }\end{array}$ & - & 6.00 & 1.98 & & & & & & & - & $.57^{*}$ & $.58^{*}$ & $.46^{*}$ \\
\hline $\begin{array}{l}\text { 9. Tiempo que } \\
\text { permanece en } \\
\text { Facebook }\end{array}$ & - & 4.38 & 2.29 & & & & & & & & - & $.52^{*}$ & $.36^{*}$ \\
\hline $\begin{array}{l}\text { 10. Cada cuánto } \\
\text { tiempo comenta el } \\
\text { perfil de sus amigos o } \\
\text { páginas }\end{array}$ & - & 4.64 & 2.31 & & & & & & & & & - & $.56^{*}$ \\
\hline $\begin{array}{l}\text { 11. Cada cuánto } \\
\text { tiempo actualiza su } \\
\text { perfil de Facebook }\end{array}$ & - & 4.15 & 2.14 & & & & & & & & & & - \\
\hline
\end{tabular}

Estas variables fueron introducidas en una regresión jerárquica con el método stepwise para determinar los predictores del auto-concepto social, el modelo final explica un $31 \%$ de la varianza total $\left(F_{3,145}=21.28, p<.001\right)$, teniéndose como principal predictor la satisfacción percibida con las relaciones interpersonales $(\beta=.36, p<.001)$, seguido de las habilidades sociales $(\beta=.23, p=.003)$ y de la cantidad de tiempo que se permanece en Facebook $(\beta=.16, p=.028)$, de tal forma que a mayor satisfacción percibida con las relaciones interpersonales, mayores habilidades sociales y más tiempo se permanezca en Facebook se puede predecir un mayor auto-concepto social (Tabla 2). 
Tabla 2. Resumen de regresión jerárquica para las variables que predicen el auto-concepto social en adolescentes $(n=148)$.

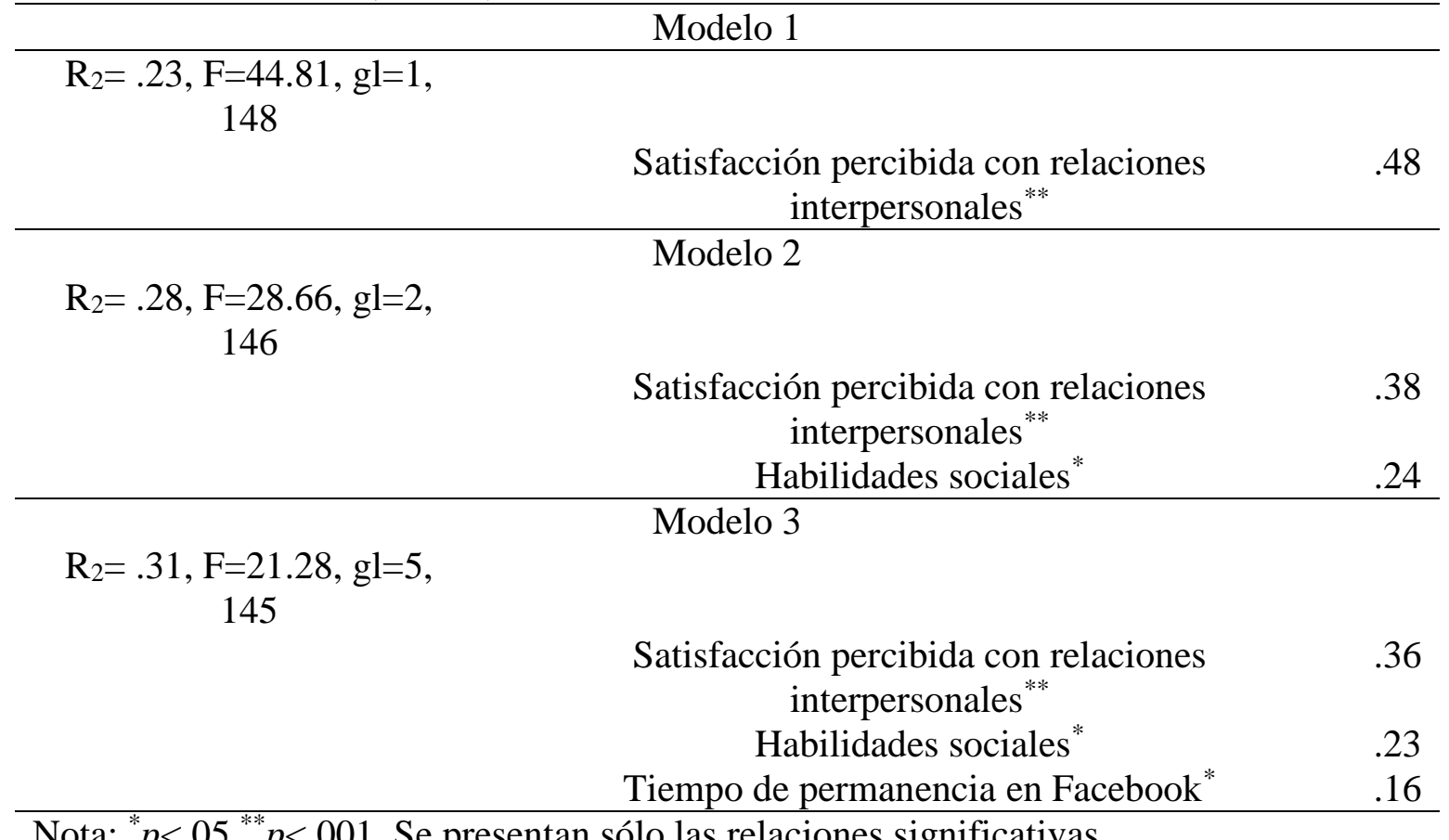

\section{Discusión}

Los resultados muestran evidencias que apoyan la hipótesis 1 planteada en el presente estudio, teniéndose así que altos niveles de satisfacción percibida con las relaciones interpersonales mediadas por computadora $\mathrm{y}$ altas habilidades sociales percibidas se relacionan con un mayor nivel de auto-concepto social. De Igual forma, se encontró una relación entre la intensidad de uso de Facebook con la interacción entre las variables antes mencionadas, dando así evidencias que apoyan a la segunda hipótesis planteada, la cual afirma que existen diferencias entre altos y bajos usuarios de Facebook con respecto a la 


\section{Uso de Facebook y Auto-concepto Social}

relación entre la satisfacción con las relaciones interpersonales, habilidades sociales y autoconcepto social.

Por otro lado, no se encontraron evidencias que respalden las hipótesis 3 (existen diferencias por sexo con respecto a la relación entre la satisfacción con las relaciones interpersonales, habilidades sociales y auto-concepto social) y 4 (la cantidad de amigos conocidos cara a cara tiene una influencia en las relaciones mencionadas anteriormente).

Las implicaciones de estos resultados serán discutidas más adelante.

\section{Estudio 2: Intensidad de uso de Facebook y su relación con el auto-concepto social en estudiantes universitarios}

\section{Método}

\section{Participantes}

Para este segundo estudio se contó con una muestra de 225 participantes con edades entre los 18 y 31 años $(M=22.03, D E=2.97)$, de los cuales 109 eran mujeres y 116 hombres, además 117 estudiaban en universidades públicas y 108 en privadas.

\section{Instrumentos}

Se utilizaron los mismos instrumentos que en el estudio 1.

\section{Procedimiento}

Como un primer paso, para comprobar la validez de los instrumentos se realizó una prueba piloto con 80 estudiantes universitarios. Luego, la recolección de datos definitiva se realizó con cuestionarios de papel y lápiz y su llenado fue de forma voluntaria. 


\section{Resultados}

Un análisis de correlación simple para determinar las relaciones entre el autoconcepto social y las demás variables en estudio mostró relaciones positivas entre el autoconcepto social y las habilidades sociales $(r=.34, p<.001)$, la satisfacción percibida con las relaciones interpersonales $(r=.44, p<.001)$, y cada cuanto tiempo comenta los perfiles de sus amigos (as) o las páginas a las que se encuentran asociados (as) $(r=.15, p=.028)$. No se encontraron relaciones significativas con el sexo $(r=.02, p=.69)$, la edad $(r=.-.17, p=.808)$, la cantidad de amigos y amigas que se tiene en Facebook $(r=.06, p=.369)$, la cantidad de amigos que conoce personalmente $(r=.10, p=.163)$, cada cuanto tiempo ingresa a Facebook $(r=.07$, $p=.338)$, el tiempo que se permanece en Facebook $(r=.08, p=.261)$, ni la cantidad de veces que actualiza su perfil de Facebook ( $r=.02, p=.749)$ (Tabla 3).

Luego, estas variables fueron introducidas en una regresión jerárquica con el método stepwise para determinar los predictores del auto-concepto social, el modelo final explica un $23 \%$ de la varianza total $(\mathrm{F} 2,210=31.61, \mathrm{p}<.001)$, teniéndose como principal predictor la satisfacción percibida con las relaciones interpersonales $(\beta=.37, \mathrm{p}<.001)$, seguido de las habilidades sociales $(\beta=.20, \mathrm{p}=.003)$, de tal forma que a mayor satisfacción percibida con las relaciones interpersonales y mayores habilidades sociales se puede predecir un mayor auto-concepto social (Tabla 4). 
Tabla 3. Medias, desviaciones típicas y correlaciones simples de las variables en estudio en estudiantes universitarios.

\begin{tabular}{|c|c|c|c|c|c|c|c|c|c|c|c|c|c|}
\hline & $\alpha$ & $\mathrm{M}$ & $\mathrm{DE}$ & 2 & 3 & 4 & 5 & 6 & 7 & 8 & 9 & 10 & 11 \\
\hline 1. Auto-concepto social & .83 & 5.03 & 1.28 & $.34^{* *}$ & $.44^{* *}$ & -.02 & .03 & .06 & .10 & .07 & .08 & $.15^{*}$ & .09 \\
\hline 2. Habilidades sociales & .76 & 5.10 & .92 & - & $.37^{* *}$ & $.17^{-}$ & .08 & .10 & $.26^{* *}$ & $.20^{* *}$ & .06 & .12 & $.16^{*}$ \\
\hline 3. Satisfacción percibida & .77 & 5.09 & .83 & & - & -.01 & $.14^{*}$ & .04 & $.25^{* *}$ & $.16^{*}$ & .08 & $.15^{*}$ & $.19^{* *}$ \\
\hline 4. Edad & - & 22.03 & 2.97 & & & - & $\begin{array}{c}- \\
.12\end{array}$ & $\begin{array}{c}- \\
.20^{* *}\end{array}$ & $\begin{array}{c}- \\
.16^{*}\end{array}$ & -.12 & -.08 & -.13 & -.11 \\
\hline 5. Sexo & - & - & - & & & & - & .07 & .06 & -.02 & -.03 & .06 & .03 \\
\hline 6. Amigos en Facebook & - & 370.40 & 397.25 & & & & & - & $.47^{* *}$ & $.29^{* *}$ & $.18^{* *}$ & $.19^{* *}$ & $.15^{*}$ \\
\hline $\begin{array}{l}\text { 7. Amigos que conoce } \\
\text { personalmente }\end{array}$ & - & 235.32 & 177.16 & & & & & & - & $.25^{* *}$ & $.16^{*}$ & $.14^{*}$ & $.14^{*}$ \\
\hline $\begin{array}{l}\text { 8. Frecuencia que ingresa } \\
\text { a Facebook }\end{array}$ & - & 5.31 & 2.11 & & & & & & & - & $.52^{* *}$ & $.46^{* *}$ & $.50^{* *}$ \\
\hline $\begin{array}{l}\text { 9. Tiempo de } \\
\text { permanencia en } \\
\text { Facebook }\end{array}$ & - & 3.74 & 1.92 & & & & & & & & - & $.41^{* *}$ & $.43^{* *}$ \\
\hline $\begin{array}{l}\text { 10. Cada cuánto tiempo } \\
\text { comenta el perfil de sus } \\
\text { amigos o páginas }\end{array}$ & - & 3.75 & 2.07 & & & & & & & & & - & $.64^{* *}$ \\
\hline $\begin{array}{l}\text { 11. Cada cuánto tiempo } \\
\text { actualiza su perfil de } \\
\text { Facebook }\end{array}$ & - & 3.03 & 1.81 & & & & & & & & & & - \\
\hline
\end{tabular}

Tabla 4. Resumen de regresión jerárquica para las variables que predicen el autoconcepto social en estudiantes universitarios $(n=212)$.

\begin{tabular}{ccc}
\hline & Modelo 1 \\
\hline $\mathrm{R}_{2}=.20, \mathrm{~F}=51.80, \mathrm{gl}=1$, & \\
211 & $\begin{array}{c}\text { Satisfacción percibida con relaciones } \\
\text { interpersonales** }\end{array}$ & .44 \\
$\beta$ & Modelo 2
\end{tabular}




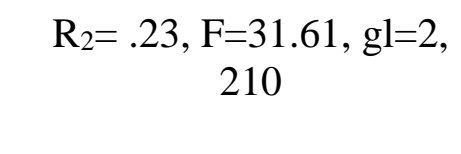

Satisfacción percibida con relaciones interpersonales $^{* *}$ Habilidades sociales*

Nota: ${ }^{*} p<.05{ }^{* *} p<.001$. Se presentan sólo las relaciones significativas.

\section{Discusión}

Los resultados muestran evidencias que apoyan la hipótesis 1 planteada en el presente estudio, teniéndose así que altos niveles de satisfacción percibida con las relaciones interpersonales mediadas por computadora y altas habilidades sociales percibidas se relacionan con un mayor nivel de auto-concepto social.

Por otro lado, no se encontraron evidencias que respalden las hipótesis 2 (existen diferencias entre altos y bajos usuarios de Facebook con respecto a la relación entre la satisfacción con las relaciones interpersonales, habilidades sociales y auto-concepto social ), 3 (existen diferencias por sexo con respecto a relación entre la satisfacción con las relaciones interpersonales, habilidades sociales y auto-concepto social) y 4 (la cantidad de amigos conocidos cara a cara tiene una influencia en las relaciones mencionadas anteriormente).

Las implicaciones de dichos resultados serán discutidas a continuación. 


\section{Discusión general}

Si bien la relación existente entre habilidades sociales, satisfacción con las relaciones interpersonales y auto-concepto social puede resultar lógica es importante notar que en el contexto de la comunicación mediada por computadora y de las redes sociales digitales estas variables han sido relacionadas de forma negativa en diversas investigaciones, proponiendo que son las personas con mayores niveles de timidez social las que permanecen más en Facebook (Orr et al., 2009) o que las personas con mayores niveles de narcisismo y con menores niveles de auto-estima son las que reportan mayor actividad en Facebook (Mehdizadeh, 2010). Sin embargo, los resultados de la presente investigación dan evidencias favorables a la hipótesis "del rico se hace más rico" (Valkenburg \& Peter, 2007a), encontrándose que son los más habilidosos socialmente los que hacen un mayor uso de las redes sociales y que el uso de estas favorecerá a su vez el auto-concepto social y sus relaciones interpersonales.

Sin embargo, la relación con el uso de Facebook, las habilidades sociales, la satisfacción percibida con las relaciones interpersonales y el auto-concepto social sólo se encontró en los adolescentes y no en los estudiantes universitarios, esto podría explicarse por la importancia que tiene el establecimiento de relaciones de amistad en la conformación de la propia identidad y por ende la moderación de variables como el apoyo social y el autoconcepto en la misma (González, Cuéllar, Miguel, \& Desfilis, 2009). De igual forma en la adolescencia se torna importante en la conformación de la propia identidad el sentido de pertenencia (Jensen, Ortiz, \& Santiago, 2008) y al ser las redes sociales un elemento novedoso del que ser parte se puede considerar una moda, el formar parte de la misma y 
participar activamente en ella puede relacionarse con una mayor identificación con el propio grupo y como consecuencia provoque un aumento en el auto-concepto social, sin embargo esta es una relación que debería de ser analizada en futuras investigaciones.

Ahora bien, el hecho de que en los estudiantes universitarios no exista una relación entre el uso de Facebook y el auto-concepto social puede deberse a que estos usuarios tienden a utilizar las redes sociales para ponerse en contacto con personas conocidas, como una moda, pasatiempo, para la búsqueda de afecto y obtener información social (noticias, reuniones sociales) más que para buscar nuevas amistades (Quan-Haase, 2008; Raacke \& BondsRaacke, 2008; Sheldon, 2008b) siendo una variable importante acá los usos que se hagan de la red social, sin embargo este es un punto que también debería de ser estudiado en el futuro.

Un punto interesante de analizar es el hecho de que la cantidad de amigos en línea no muestra una relación con el auto-concepto social, punto que podría explicarse hipotetizando que el auto-concepto social no necesariamente esté ligado a la cantidad de amigos que se posea si no al nivel de satisfacción que se tiene con respecto a las relaciones que se tienen, variable que si se relaciona en ambos grupos con el auto-concepto social. Este sería un aspecto relevante de estudiar con análisis de trayectorias determinando si la cantidad de amigos guarda una relación con la satisfacción percibida con las relaciones interpersonales y esta a su vez promueve el auto-concepto social.

Una limitación del presente estudio es el hecho de que la intensidad de uso de Facebook se realizó por medio de preguntas tipo Likert y quizá sería más beneficioso 
realizarlo a través de una medición más exacta como un horario o la cantidad de horas que se dedica a navegar por esta red.

\section{Referencias}

Brenes, C. (2009). Dimensiones psicosociales asociadas al uso de los video juegos en niños y niñas escolares de instituciones públicas y privadas de zonas urbanas de San José. Tesis para optar por el grado de Licenciatura en Psicología. Universidad de Costa Rica. Facultad de Ciencias Sociales, Escuela de Psicología.

Chan, D. K., \& Cheng, G. H. (2004). A Comparison of Offline and Online Friendship Qualities at Different Stages of Relationship Development. Journal of Social and Personal Relationships, 21, 305-320.

Gangadharbatla, H. (2008). Facebook Me: Collective Self-Esteem, Need to Belong,and Internet Self-Efficacy as Predictors of the iGeneration's Attitudes toward Social Networking Sites. Journal of Interactive Advertising, 8(2), 5-15.

Gonzales, A. L., \& Hancock, J. T. (2011). Mirror, Mirror on my Facebook Wall: Effects of Exposure to Facebook on Self-Steem. Cyberpsychology, Behavior and Social Networking, 14(1-2), 79-83.

González, J. J. Z., Cuéllar, A. I., Miguel, J. M. T., \& Desfilis, E. S. (2009). El desarrollo de la identidad en la adolescencia y adultez emergente: Una comparación de la identidad global frente a la identidad en dominios específicos. Anales de psicología, 25(2), 316-329. 
Jensen, J., Ortiz, M. E., \& Santiago, Y. (2008). Adolescencia y adultez emergente: Un enfoque cultural: Pearson Educación.

Joinson, A. (2001). Self-Disclosure on Computer Mediated Communication: The role of self-awareness and visual anonymity. European Journal of Social Psychology, 31, $177-192$.

Kujath, C. L. (2011). Facebook or MySpace: Complement or Substitute for Face-to-Face Interaction? Cyberpsychology, Behavior and Social Networking, 14(1-2), 75-78.

Ledbetter, A. M., Mazer, J. P., DeGroot, J. M., Meyer, K. R., Mao, Y., \& Swafford, B. (2011). Attitudes Towards Online Social Connection and Self-Disclosure as Predictors of Facebook Communication and Relational Closeness. Communication Research, 38(1), 27-53.

Mehdizadeh, S. (2010). Self-Presentation 2.0: Narcissism and Self-Esteem on Facebook. Cyberpsychology, Behavior and Social Networking, 13(4), 357-364.

Nielsen. (2010). Social Networks/Blogs Now Account for One in Every Four and a Half Minutes Online, from http://blog.nielsen.com/nielsenwire/online_mobile/socialmedia-accounts-for-22-percent-of-time-online/

Orr, E. S., Sisic, M., Ross, C., Simmering, M. G., Arseneault, J. M., \& Rorr, R. R. (2009). The Influence of Shyness on the Use of Facebook in an Undergraduate Sample. CyberPsychology and Behavior, 12(3), 337-340.

Quan-Haase, A. (2008). Instant Messaging on Campus: Use and Integration in University Student's Everyday Communication. The Information Society, 24, 105-115. 
Quan-Haase, A., \& Young, A. L. (2010). Uses and Gratifications of Social Media: A

Comparison of Facebook and Instant Messaging. Bulletin of Science Technology \& Society, 30(5), 350-361.

Raacke, J., \& Bonds-Raacke, J. (2008). MySpace and Facebook: Applying the Uses and Gratifications Theory to Exploring Friend-Networking Sites. CyberPsychology and Behavior, 11(2), 169-174.

Sassenberg, K., Boos, M., \& Radung, S. (2005). Attitude change in face-to-face and computer-mediated communication: private self-awareness as mediator and moderator. European Journal of Social Psychology, 31, 361-374.

Sheeks, M., \& Birchmeier, Z. (2007). Shyness, Sociability, and the Use of ComputerMediated Communication in Relationship Development. CyberPsychology and Behavior, 10, 64-70.

Sheldon, P. (2008a). The Relations Between Unwillingness-to-Communicate and Student's Facebook Use. Journal of Media Psychology, 20(2), 67-75.

Sheldon, P. (2008b). Student Favorite: Facebook and Motives for its Use. Southwestern Mass Communication Journal, 23, 39-54.

Socialbakers. (2012). Costa Rica Facebook Statistics, from http://www.socialbakers.com/facebook-statistics/costa-rica

Torres, D. (2009). Prevalencia de conductas socialmente habilidosas en hombres jóvenes universitarios heterosexuales al iniciar conversaciones con personas del sexo opuesto en situaciones de cortejo y si correlación con las variables asertividad, 
auto-eficacia y auto-concepto (Tesis de Licenciatura sin publicar). Universidad de Costa Rica, San José.

Valkenburg, P. M., \& Peter, J. (2007a). Adolescents' online communication and their wellbeing: Testing the stimulation versus the displacement hypothesis. Journal of Computer mediated communication, 12 (4), article 2.

Valkenburg, P. M., \& Peter, J. (2007b). Internet Communication and Its Relation to WellBeing: Identifying Some Underlying Mechanisms. Media Psychology, 9, 43-58. Valkenburg, P. M., Peter, J., \& Schouten, A. (2006). Friend networking sites and their relationship to adolescents' well-being and social self-esteem. CyberPsychology and Behavior, 9, 585-590.

Van-der-Bergh, B. R. H., \& Marcoen, A. (1999). Harter's Self-Perception Profile for Children: Factor structure, reliability, and convergent validity in a Dutch-speaking Belgian sample of fourth, fifth and sixth graders Psychologica Belgica, 39(1), 2947.

Vindas, L. (2010, 22 de agosto). Redes sociales son usadas por el 95\% de usuarios web de Costa Rica, El Financiero. Retrieved from http://www.elfinancierocr.com/ef_archivo/2010/agosto/22/tecnologia2487734.html

Wise, K., Alhabash, S., \& Park, H. (2010). Emotional Responses During Social Information Seeking on Facebook. Cyberpsychology, Behavior and Social Networking, 13(5), 555-562. 